\title{
Study on Simple Signal Area Estimation for Efficient Spectrum Measurements
}

\author{
Kenta Umebayashi $\dagger$, and Hiroki Iwata \\ Dept. of Electrical \& Electronic Eng., \\ Tokyo University of Agriculture and Technology, \\ Japan, Email: ume_k@cc.tuat.ac.jp $\dagger$
}

\author{
Janne J. Lehtomäki \\ Centre for Wireless Communications, \\ University of Oulu, \\ Finland
}

\author{
Miguel López-Benítez \\ Dept. of Electrical \\ Eng. \& Electronics, \\ University of Liverpool, UK
}

\begin{abstract}
This paper investigates a signal area (SA) estimation method for spectrum measurements. The spectrum measurements is for estimating statistical information of spectrum usage and required latency is not very critical compared to spectrum sensing. SA denotes the area (in time/frequency domain) occupied by the primary user's signal. The traditional approach, which utilizes Fourier transform (FT) and energy detector (ED) for SA estimation, can achieve low complexity, but its estimation performance is not very high. For this issue, we propose a postprocessing, simple SA (S-SA) estimation. S-SA estimation exploits the correlation of the spectrum states among the neighboring tiles, i.e., time/frequency grids, and the fact that SA typically has a rectangular shape to estimate $S A$ with high accuracy and relatively low complexity compared to a conventional method, contour tracing based SA (CT-SA) estimation. Numerical results will show that the S-SA estimation method can achieve better detection performance than CT-SA. Furthermore, it can reduce the computation time compared to the CT-SA estimation.
\end{abstract}

\section{INTRODUCTION}

In the wireless communication field, spectrum scarcity problem is a pressing problem. On the other hand, spectrum utilization measurement reports have revealed that the utilization rate of most of the licensed spectrum is not very high in spatial and/or temporal domains. The portions of spectrum left unused are commonly referred to as white space (WS) [1]. For this problem, dynamic spectrum access (DSA) with cognitive radio techniques employed by the unlicensed user (secondary user: SU) on the spectrum owned by the licensed user (primary user: PU) have been investigated [2]. In the DSA, the SU can utilize the vacant spectrum as long as it will not cause any harmful interference to the PU. To enable DSA, a technique for understanding the state of the spectrum, i.e. whether it is vacant or occupied, is necessary. Spectrum sensing [3] techniques have been investigated in order to understand the instantaneous spectrum state. However, it requires high accuracy, low latency and low cost and it is difficult achieve the all requirements. One potential approach to solve the issue in spectrum sensing is smart spectrum access (SSA) which is an extended DSA that utilizes useful prior information, such as statistical information of PU spectrum usages [4]. The statistical information can enhance not only spectrum sensing [5], but also other techniques, such as spectrum management, channel selection and MAC protocol design [6], [7]. One important issue in SSA is the implementation cost of the function obtaining the prior information, especially at wireless terminals. For this issue, we proposed a system architecture consisting of two layers in [4]: the first layer corresponds to a DSA system and the second layer is a spectrum awareness system (SAS). In the twolayer SSA, the SAS is dedicated for spectrum measurement to obtain the prior information. Therefore, DSA terminals no longer suffer from the high implementation cost, but the useful information is provided by the SAS.

In this paper, we focus on spectrum usage measurements at observation equipments (OEs) in SAS and the purpose of spectrum measurements is to obtain the statistical information. There are several spectrum measurement campaigns for the assessment of WS based on spectrum usage detection [8], [9]. One of the basic approaches in the spectrum measurements is FT (Fourier transform)-based energy detector (ED) to detect PU spectrum utilization in the considered frequency bins and is denoted by FT-ED. Output of the FT-ED based spectrum measurement consists of signal detection results in two-dimensional time/frequency grids. Let us denote a single element of the grid corresponding to one time slot and one frequency bin by tile. In addition, a rectangular set of contiguous tiles wherein a PU signal is detected is referred to as estimated signal area (SA). Detection result $\hat{H}$ can be either $H_{1}$ (the tile is occupied by PU signal) or $H_{0}$ (the tile is not occupied). In signal detection, there are two errors: the first one is missed detection $\left(\hat{H}=H_{0} \mid H_{1}\right)$ where $\mid$ means given that correct hypothesis is $H_{1}$, and the second one is false alarm $\left(\hat{H}=H_{1} \mid H_{0}\right)$. ED is very simple and does not require any prior information about the PU signal [10], however the detection performance is not very high [3].

A post-processing to the outputs of FT-ED can easily improve the detection performance and this is applicable in the spectrum measurement for obtaining statistical information. Specifically, latency requirements in spectrum measurement are relaxed compared to spectrum sensing. Now we define SA as follows: a cluster of $H_{1}$ tiles due to one continuous signal transmission such as one data packet. The time duration of SA is determined by the time duration of the continuous signal and the bandwidth of the SA is determined by the symbol rate and band limited filter used in the transmitter. SA has typically a rectangular shape [11], since symbol rate is typically fixed during one continuous signal. There are several methods employing the post-processing [12], [13]. In [14], FT-ED is used and clusters of connected $\hat{H}=H_{1}$ tiles are obtained by using standard contour tracing (CT) techniques [15]. This approach is denoted by CT-SA and corresponds to a baseline for comparisons with our proposed method.

In this paper, we propose an SA estimation based on the output of the Welch FFT based ED. Welch FFT [16] based ED 
can achieve better detection performance compared to typical FFT based ED [17]. Our main contributions in this paper are summarized as follows:

- $\quad$ Simple-SA (S-SA) estimation is proposed in this paper. Unlike conventional CT-SA estimation, S-SA estimates the width corresponding to frequency domain and the height corresponding to time domain of the rectangle which leads to less complexity. Similar to CT-SA, S-SA combines the several detection results in time and frequency domains and it can recognize SA accurately.

- Extensive performance evaluations are performed to verify the performances and complexity benefits of SSA estimation compared with the FT-ED and CT-SA.

\section{Outline of Signal Processing in ObSERVATion EQUIPMENT (OE)}

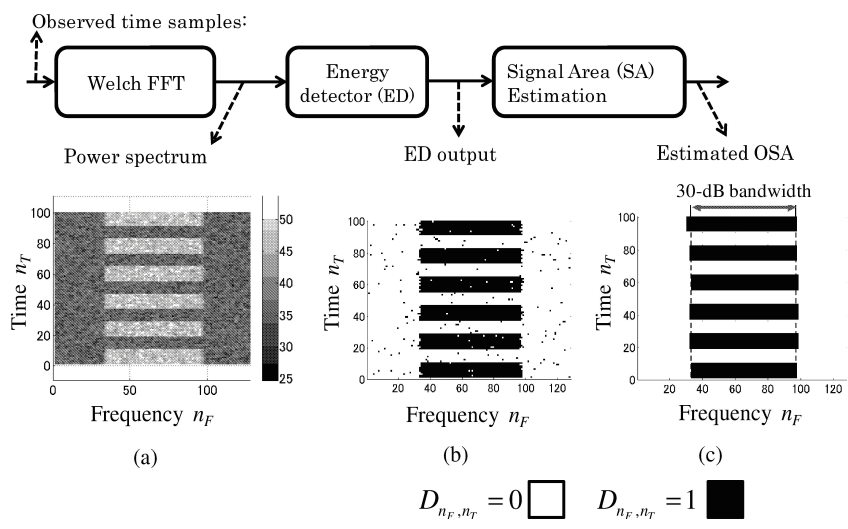

Fig. 1: Block diagram for spectrum measurement in OE.

A block diagram of the signal processing flow in $\mathrm{OE}$ is shown in Fig. 1. The signal processing in the OE consists of three processes; Welch FFT, ED, and SA estimation. The details about ED based on Welch FFT are provided in our earlier paper [17].

Observed samples are obtained at $f_{S} \mathrm{~Hz}$ sampling rate. The center frequency of the observed spectrum is set to $0 \mathrm{~Hz}$ by downconversion, and it is band-limited to $\left[-f_{S} / 2, f_{S} / 2\right]$ $\mathrm{Hz}$. The observed bandwidth is large enough compared to the PU signal bandwidth. We assume AWGN channel, i.e., nonfrequency selectivity and signal power is constant during the measurement.

To obtain PSD, Welch FFT is used and the number of samples in time domain for single Welch FFT is denoted by $N_{S}$. The $N_{S}$ samples resulting from the Welch FFT correspond to a single time slot. We also set the number of time slots in one observation to $N_{\text {timeslot}}$. In the Welch FFT, $N_{S}$ samples are divided into $N_{\text {seg }}$ segments where the number of samples in one segment is denoted by $N_{W}$. Without loss of generality, $N_{S}$ and $N_{W}$ are assumed to be powers of two and $N_{s e g}$ is given by

$$
N_{\text {seg }}=\frac{N_{S}-N_{W}}{N_{O}}+1=\frac{2 N_{S}}{N_{W}}-1,
$$

where $N_{O}$ indicates overlapped samples which is set to $N_{O}=N_{W} / 2$ and $N_{S}>N_{W}$. The obtained PSD values are denoted by $P_{n_{F}, n_{T}}$ where $n_{F} \in\left\{1,2, \ldots, N_{\mathrm{W}}\right\}$ and $n_{T} \in\left\{1,2, N_{\text {timeslot }}\right\}$ are the frequency and time index numbers of the tile. The Fig. 1 (a) shows an example of the PSD values. In the Welch FFT, Hamming window is used.

The ED provides signal detection result at the tile $\left(n_{F}, n_{T}\right)$ and it is given by

$$
D_{n_{F}, n_{T}}^{(\mathrm{ED})}= \begin{cases}1 & \left(P_{n_{F}, n_{T}}>\tau\right) \\ 0 & \text { (otherwise) }\end{cases}
$$

where $D_{n_{F}, n_{T}}^{(\cdot)}=1$ and $D_{n_{F}, n_{T}}^{(\cdot)}=0$ indicate that the tile is either estimated to be occupied by PU signal or vacant, respectively, the superscript notation $(\cdot)$ indicates the method to obtain the decision, such as S-SA estimation, and $\tau$ is the threshold for ED. Fig. 1 (b) shows an example of $D_{n_{F}, n_{T}}^{(\mathrm{ED})}$.

There are several ways to define the bins where signal is actually present. We use the $30-\mathrm{dB}$ bandwidth [18], so that the signal bandwidth is defined by the frequency bins when the signal power is $30 \mathrm{~dB}$ below its peak value. The corresponding $\mathrm{SA}$ is defined by the signal bandwidth and the time duration of the continuous signal emission. A correct decision for a frequency bin within this area is $H_{1}$ otherwise $H_{0}$ is correct.

We define false alarm probability for the output of ED as $\mathrm{P}_{\mathrm{FA}}{ }^{(\mathrm{ED})}=\operatorname{Pr}\left(D_{n_{F}, n_{T}}^{(\mathrm{ED})}=1 \mid H\left(n_{F}, n_{T}\right)=H_{0}\right)$ where $H\left(n_{F}, n_{T}\right)$ indicate the spectrum state in the tile at $\left(n_{F}, n_{T}\right)$ and $H_{0}$ indicates that the tile is not occupied by PU signal. In addition, detection probability $\left(\mathrm{P}_{\mathrm{D}}{ }^{(\mathrm{ED})}\right)$ indicates that $\mathrm{P}_{\mathrm{D}}{ }^{(\mathrm{ED})}=\operatorname{Pr}\left(D_{n_{F}, n_{T}}^{(\mathrm{ED})}=1 \mid H\left(n_{F}, n_{T}\right)=H_{1}\right)$ where $H_{1}$ indicates that the tile is occupied by PU signal.

All the SA estimation approaches utilize the outputs from ED and process them. Due to processing, the false alarm probability in the output may change compared to the false alarm probability in the input data. Thus in SA estimation, there are two false alarm probabilities, such as $\mathrm{P}_{\mathrm{FA}}{ }^{(\mathrm{ED})}$ and $\mathrm{P}_{\mathrm{FA}}{ }^{(\mathrm{S}-\mathrm{SA})}$. The threshold $\tau$ is set based on target false alarm rate $\tilde{\mathrm{P}}_{\mathrm{FA}}^{(\mathrm{S}-\mathrm{SA})}$, where $\sim$ indicates target value.

The description of general behavior of SA estimation shown in (b) and (c) of Fig. 1 is described as follows. In Fig. 1 (b), $D_{n_{F}, n_{T}}^{(\mathrm{ED})}$ (ED output) are clusters colored by dark color and they are almost rectangular. The SA estimation recognizes the clusters and approximates them to rectangles as shown in Fig. 1 (c). The white colored tiles $\left(D_{n_{F}, n_{T}}^{(\mathrm{ED})}=0\right)$ inside the rectangles in Fig. 1 (b) are missed detections. The SA estimation has a potential to reduce the missed detections by filling the white colored tiles within rectangle.

\section{Signal AREA Estimation}

The general outline of the process common to both CTSA and S-SA estimation methods is illustrated in Fig. 2. In both SA estimation methods, the first step of the algorithm is to find a starting point for the occupied signal area. This is done by performing a raster scan on the spectrum grid from left to right and bottom to up until the first occupied tile, i.e. $D_{n_{F}, n_{T}}^{(\mathrm{ED})}=1$, is found. The coordinates of the first tile are denoted by $\mathbf{S}$ and in the example of Fig. 2 (a) are equal to $\mathbf{S}=(3,2)$. After this, each method estimates the 


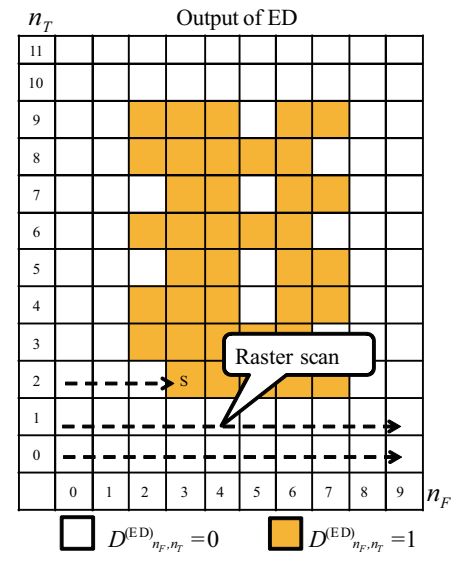

(a)

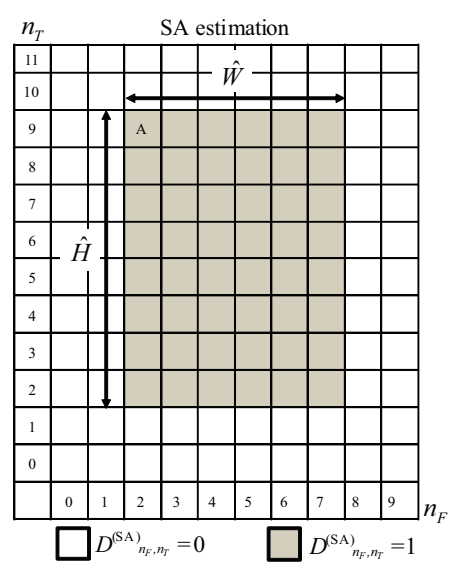

(b)
Fig. 2: Example outputs of ED and SA estimation.

occupied signal area, and provides an estimation result as a rectangle which covers the area as shown in Fig. 2-(b). The output of the estimation is denoted by estimated rectangle SA in this paper. In the SA approach, the estimation result can be represented with a corner coordinate, and the width and height of the rectangle, such as $\mathrm{A}, \hat{W}$, and $\hat{H}$ in Fig. 2-(b). However, the spectrum grid obtained with an ED represents signal occupancy information in the observed time-frequency space. Therefore, in wideband or long-term measurements rectangular signal area estimate has the benefit of reducing the amount of required information. In our implementation of the CT-SA [14], we used the standard CT technique [15] which can obtain the cluster of connected tiles with $D_{n_{F}, n_{T}}^{(\mathrm{ED})}=1$. The estimated rectangle $\mathrm{SA}$ corresponds to the minimum rectangle which can cover the cluster.

Details of the successive process after the raster scan in the S-SA estimation are described in the following sub-section.

The difference to the CT-SA method is that since S-SA is designed to estimate directly a rectangular area, on the other hand CT technique is designed to find arbitrary shape. Therefore, S-SA is generally simpler. Furthermore, since the estimation is performed using larger detection masks covering multiple tiles, it has the benefit of combining multiple masks and covering small gaps between tiles. With proper mask size, it is possible to improve SA estimation performance due to diversity gain by combining multiple ED decisions.

\section{A. Simple SA Estimation}

After finding the starting point $\mathbf{S}$, the S-SA method consists of three separate steps: width estimation (step 1), coarse height estimation (step 2) and fine height estimation (step 3).

The first step of the S-SA method is the width estimation and the process is shown in Fig. 3. We set a parameter $\Delta t$ which determines size of the detection mask used to detect signal area in the first step. Starting from $\mathbf{S}=\left(x_{s}, y_{s}\right)$ the algorithm checks the detected occupancy of tiles in $n_{F}=x_{s}$ and $y_{s} \leq n_{T} \leq y_{s}+\Delta t$. If one or more of these tiles are determined as occupied, the algorithm moves to the right to $n_{F}=x_{s}+1$ and repeats the process. The right edge is found

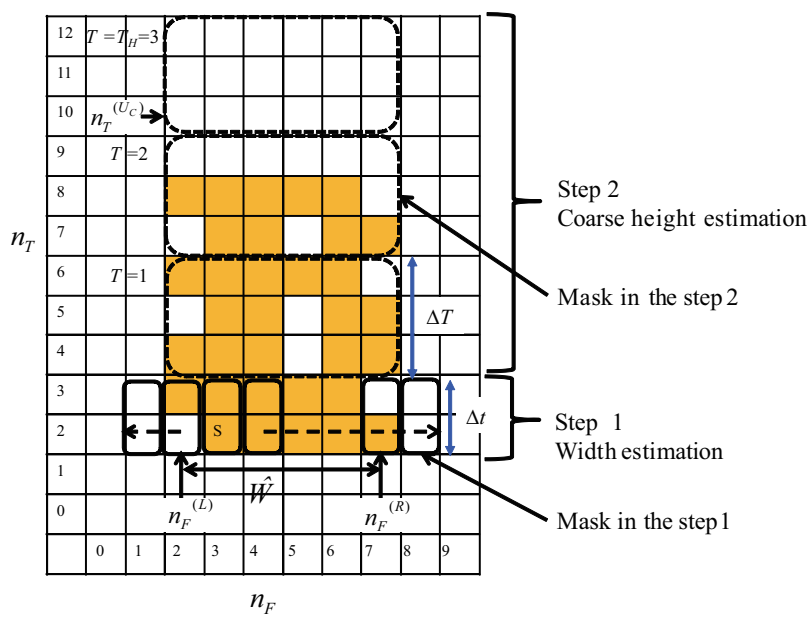

Fig. 3: Process of S-SA step 1 and step 2.

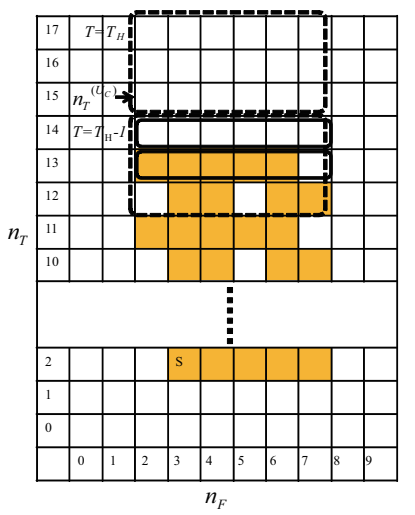

(a)

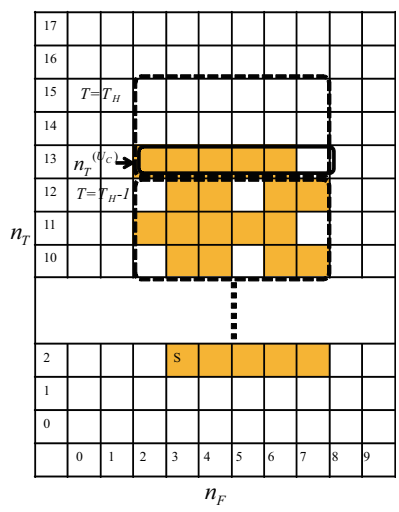

(b)
Fig. 4: Process of S-SA step 3.

when all the tiles inspected are detected as unoccupied. The left edge can be found correspondingly by moving to the opposite direction from the starting point $\mathbf{S}$. The left and right edge estimates are found as $n_{F}^{(L)}$ and $n_{F}^{(R)}$, respectively, which then provides the width estimate as $\hat{W}=n_{F}^{(R)}-n_{F}^{(L)}+1$. In the example of Fig. 3, $n_{F}^{(L)}=2, n_{F}^{(R)}=7$ and $\hat{W}=6$.

Next step provides a rough estimate for the height of the rectangle and an example can also be found in Fig. 3. Parameter $\Delta T$ is set which denotes the height of the detection mask and the width of the mask is set to $\hat{W}$. Threshold controlling the sensitivity is set to $0<\gamma_{\text {step2 }}<1$. The detection mask begins with its bottom row at time slot $x_{s}+\Delta t+1$. The algorithm then checks the occupancy of the tiles covered by the occupied and if more than $\gamma_{\text {step } 2} \times \hat{W} \Delta T$ tiles are detected as occupied, the area covered by the mask is determined as SA. This condition is denoted by sensitivity condition. The mask is then moved up by $\Delta T$ time slots and the process is repeated until a mask not satisfying the sensitivity condition is found. The index number of mask is denoted by $T$ and $T=T_{H}$ at the first mask not satisfying the sensitivity condition. This then provides the rough estimate for the upper part of the estimated 
area as $n_{T}^{\left(U_{C}\right)}$ which corresponds to the bottom time slot in the $T_{H}$ th mask.

In the rough height estimation there are two different scenarios to consider which are illustrated in Fig. 4 (a) and (b). In the scenario (a), the algorithm stops because there are no occupied tiles in the last mask. On the other hand, in the scenario (b), the algorithm stops because of the sensitivity condition is not satisfied but there are still less than $\gamma_{\text {step } 2} \times \hat{W} \Delta T$ occupied tiles in the mask above the estimated $n_{T}^{\left(U_{C}\right)}$ time slot. Therefore, a final height estimation step is performed to obtain a more accurate result. In this fine height estimation, the detection mask is set to $\hat{W}$ frequency bins and this corresponds to setting $\Delta T=1$ in step 2 . We also set a sensitivity parameter $0<\gamma_{\text {step3 }}<1$ and check if more than $\gamma_{\text {step } 3} \times \hat{W}$ tiles under the mask are occupied or not. The search starts at $n_{T}^{\left(U_{C}\right)}$. If the sensitivity condition is not satisfied at the $n_{T}^{\left(U_{C}\right)}$ time slot, it goes downwards to find a detection mask which satisfies the sensitivity condition. This corresponds to scenario (a) in Fig. 4 and the time slot satisfying the sensitivity condition is the final estimate $n_{T}^{\left(U_{F}\right)}$.

On the other hand, if the sensitivity condition is satisfied at the $n_{T}^{\left(U_{C}\right)}$ time slot, it goes to upwards to find a detection mask which does not satisfy the sensitivity condition. This corresponds to scenario (b) in Fig. 4 and the previous time slot of the time slot not satisfying the sensitivity condition is the final estimate $n_{T}^{\left(U_{F}\right)}$. The decision result obtained by SSA is denoted by $D_{n_{F}, n_{T}}^{(\mathrm{S}-\mathrm{SA})}$ and the state of tiles inside the estimated SA is $D_{n_{F}, n_{T}}^{(\mathrm{SA}-\mathrm{n})}=1$.

\section{NumericAl EVALUATIONS}

In this section, we will evaluate SA estimation methods (CT-SA and S-SA) and the typical approach, i.e. FT-ED, in terms of detection probability, and computational time. Assumptions in the numerical evaluations are as follows. Observed bandwidth is set to $40 \mathrm{MHz}$, i.e., $f_{S}=2 B_{S}=40 \mathrm{MHz}$ and the $30-\mathrm{dB}$ bandwidth is set to $22 \mathrm{MHz}$. This bandwidth is equal to a bandwidth in IEEE 802.11g WLAN since it assumes strong filtering used at the transmitter. Number of samples in a time frame is set to $N_{S}=1024$ and the number of samples in segment size of Welch FFT to $N_{W}=128$. The number of $N_{W}$ corresponds to the number of frequency bins. The number of time slots for one observation is set to $N_{\text {timeslot }}=300$.

To fairly evaluate detection performance, we use CFAR in the evaluations of detection probability and computational time. The target false alarm rate for output of the SA methods is set to $\tilde{\mathrm{P}}_{\mathrm{FA}}^{(\mathrm{SA})}=0.01$.

We set $\Delta t$ and $\Delta T$ on the assumption that WLAN is operating in ISM band. Specifically, based on the Monte Carlo simulations we found proper parameter setting as $\Delta t=8$ and $\Delta T=4$. Moreover, $\gamma_{\text {step2 }}$ and $\gamma_{\text {step3 }}$ are set based on minimizing RMSE (root mean square error) with target SNR being equal to $-5 \mathrm{~dB}$, which is reasonable since most challenging for spectrum measurement and sensing is performing with low SNR values such as $-5 \mathrm{~dB}$, i.e. $\gamma_{\mathrm{step} 2}=0.1$ and $\gamma_{\text {step3 }}=0.15$, by the Monte Carlo simulations.

In Fig. 5, detection probability obtained by Mote Carlo simulation verse SNR is shown. According to the CFAR,

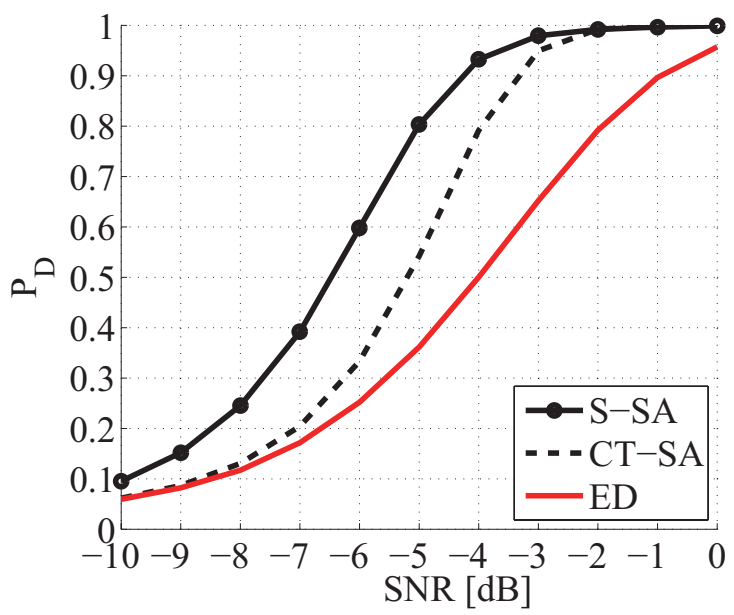

Fig. 5: Detection rate verse SNR.

false alarm rate for outputs of the SA estimation methods, and the FT-ED are set to 0.01 . We can confirm that the SSA estimation can achieve the best detection performance. Specifically, detection probability of the S-SA is better than that of CT-SA and the difference in terms of SNR for achieving $P_{D}=0.9$ is around $1 \mathrm{~dB}$. S-SA estimation can improve the overall detection probability by up to $25 \%$ with respect to CTSA and by up to $43 \%$ with respect to ED. The reason why the S-SA can achieve better performance is as follows. ED output $D_{n_{F}, n_{T}}^{(\mathrm{ED})}$ for one continuous signal transmission can be divided into several sub-clusters which indicates a cluster in which tiles with $D_{n_{F}, n_{T}}^{(\mathrm{ED})}=1$ are contiguous. The CT-SA can only recognize sub-cluster separately in most of cases. However, the S-SA can combine several sub-clusters by gap filling and this ability leads to better detection performance.

Compared to the typical approach, FT-ED, our proposed method S-SA with FC can achieve more than $4 \mathrm{~dB}$ gain in SNR for achieving $P_{D}=0.9$.

To evaluate the computational cost, we evaluate computation time for the SA estimation methods in Fig. 6. The algorithms were implemented with MATLAB. The evaluated computation time represents the time required to perform SA estimation methods for one hundred observations. In any SNR, computation times of S-SA estimation method is faster than that of CT-SA estimation method. S-SA estimation can reduce the computation time of CT-SA by up to $57 \%$. This is because S-SA estimates only width and height. In addition detection mask based estimation can reduce the computational time compared to the CT-SA which checks the state of each tile one by one.

In the high SNR region, such SNR is more than $-2 \mathrm{~dB}$, both methods can achieve $P_{D} \simeq 1$, therefore difference in terms of the computation times is not significant. On the other hand, in the region where SNR is more than $-6 \mathrm{~dB}$ and less than -2 $\mathrm{dB}$, both computation times are increased as SNR decreases. In this region, $P_{D}<1$ and each actual $\mathrm{SA}$ is divided into several estimated SAs and the number of divided estimated SAs is proportional to the computation time. In the low SNR region, such SNR is less than $-6 \mathrm{~dB}, P_{D}$ is significantly low 


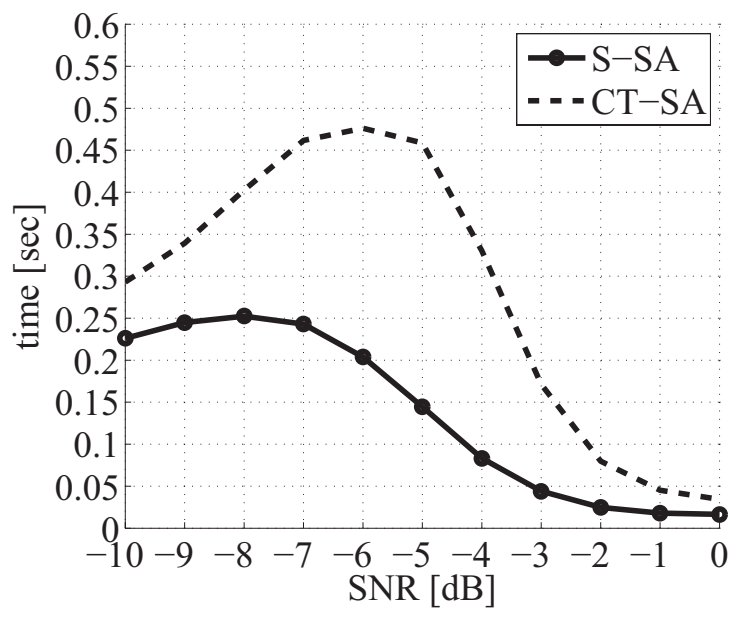

Fig. 6: Computational time as a function of SNR. Computer specifications: CPU is Intel Core i7-2600 @3.4 GHz, size of memory size is $8 \mathrm{G}$ byte, and programing language is MATLAB.

and it decreases the number of estimated SAs and leads to low computation time.

\section{CONCLUSION}

In this paper, we have investigated SA estimation techniques for spectrum measurement. To perform proper measurement, the $\mathrm{OE}$ is required to be able to achieve adequate detection accuracy at relatively low computational cost as well as low amount of information in terms of local observations. A typical approach for spectrum measurement is FT-ED, however it cannot achieve adequate detection accuracy. For this issue, we have proposed a simple SA estimation method, S-SA. The S-SA is designed to estimate rectangular SA efficiently. Specifically, the S-SA only estimates the location and the width and height and it leads to less complexity. In addition, diversity gain is available due to the covering several tiles. Therefore SA can improve detection probability and it was shown by numerical results, such as three $\mathrm{dB}$ gain in SNR for achieving 0.9 detection probability. In addition, it is also possible to achieve shorter computational time especially in the low SNR region.

\section{ACKNOWLEDGMENT}

This research and development work was supported by the MIC/SCOPE \#165003006, and JSPS KAKENHI Grant Numbers JP15K06053, JP15KK0200.

\section{REFERENCES}

[1] I. F. Akyildiz, W.-Y. Lee, M. C. Vuran, and S. Mohanty, "A survey on spectrum management in cognitive radio networks," IEEE Commun. Mag., vol. 46, no. 4, pp. 4182-4195, Apr. 2008.

[2] Q. Zhao, "A survey of dynamic spectrum access: signal processing, networking, and regulatory policy," IEEE Signal Processing Mag., vol. 24, pp. 79-89, May 2007.

[3] T. Yucek and H. Arslan, "A survey of spectrum sensing algorithms for cognitive radio applications," IEEE Communications Surveys \& Tutorials, vol. 11, pp. 116-130, 2009.
[4] K. Umebayashi, S. Tiiro, and J. J. Lehtomaki, "Development of a measurement system for spectrum awareness," in Proc. of 1st International Conference on 5G for Ubiquitous Connectivity, Nov. 2014.

[5] T. Nguyen, B. L. Mark, and Y. Ephraim, "Spectrum sensing using a hidden bivariate markov model," IEEE Trans. Wireless Commun., vol. 12, no. 9, pp. 4582-4591, Sept. 2013.

[6] K. Umebayashi, Y. Suzuki, and J. Lehtomäki, "Dynamic selection of CWmin in cognitive radio networks for protecting IEEE 802.11 primary users," in Proc. CROWNCOM, June 2011, pp. 266-270.

[7] Y. Xu, A. Anpalagan, Q. Wu, L. Shen, Z. Gao, and J. Wang, "Decisiontheoretic distributed channel selection for opportunistic spectrum access: Strategies, challenges and solutions," IEEE Communications Surveys and Tutorials, vol. 15, no. 4, pp. 1689-1713, 2013.

[8] M. Mehdawi, N. Riley, K. Paulson, A. Fanan, and M. Ammar, "Spectrum occupancy survey in hull-uk for cognitive radio applications: Measurement \& analysis," International Journal of Scientific \& Technology Research, vol. 2, no. 4, pp. 231-236, Apr. 2013.

[9] M. López-Benítez and F. Casadevall, "Spectrum usage in cognitive radio networks: From field measurements to empirical models," IEICE Trans. Commun.,, vol. E97-B, no. 2, pp. 242-250, Feb. 2014.

[10] H. Urkowitz, "Energy detection of unknown deterministic signals," Proc. IEEE, vol. 55, no. 4, pp. 523-531, Apr. 1967.

[11] J. Vartiainen, J. Lehtomaki, T. Braysy, and K. Umebayashi, "Spectrum sensing in public safety applications: The 2-d lad acc method," in Proc. Cognitive Radio Oriented Wireless Networks \& Communications (CROWNCOM), June 2011, pp. 61-65.

[12] J. Vartiainen, H. Sarvanko, J. Lehtomaki, M. Juntti, and M. LatvaAho, "Spectrum sensing with lad-based methods," in Proc. IEEE International Symposium on Personal, Indoor and Mobile Radio Communciations (PIMRC), Sept. 2007, pp. 1-5.

[13] M. López-Benítez and F. Casadevall, "Improved energy detection spectrum sensing for cognitive radio," IET Communications, vol. 6, no. 8, pp. 785-796, May 2012.

[14] J. Kokkoniemi and J. Lehtomaki, "Spectrum occupancy measurements and analysis methods on the $2.45 \mathrm{ghz}$ ISM band," in Proc. Cognitive Radio Oriented Wireless Networks \& Communications (CROWNCOM), June 2012, pp. 285-290.

[15] D. W. Capson, "Parformance comparisons of contour extraction algorithms," IEEE Trans. Instrum. Meas., vol. IM-35, no. 4, pp. 409-417, Dec. 1986.

[16] P. D. Welch, "The use of fast fourier transform for the estimation of power spectra: A method based on time averaging over short, modified periodograms," IEEE Trans. Audio Electroacoust., vol. 15, pp. 70-73, June 1967.

[17] K. Umebayashi, R. Takagi, N. Ioroi, Y. Suzuki, and J. J. Lehtomaki, "Duty cycle and noise floor estimation with welch fft for spectrum usage measurements," in Proc. of Cognitive Radio Oriented Wireless Networks and Communications (CROWNCOM), June 2014, pp. 73 78.

[18] "Recommendation ITU-R SM.328-10. Spectra and bandwidth of emissions," 1992. 